\title{
Parallel EELS elemental mapping in scanning transmission electron microscopy: use of the difference methods
}

\author{
Gérard Balossier $\left({ }^{1}\right)$, Xavier Thomas $\left({ }^{1}\right)$, Jean Michel $\left({ }^{1}\right)$, Denis Wagner $\left({ }^{1}\right)$, Pierre Bonhom- \\ me $\left({ }^{1}\right)$, Edith Puchelle $\left({ }^{2}\right)$, Dominique Ploton $\left({ }^{2}\right)$, Annie Bonhomme $\left({ }^{2}\right)$ and Jean Michel \\ Pinon $\left(^{2}\right)$ \\ (1) Laboratoire de Microscopie Electronique, Unité INSERM 314, 21 rue Clément Ader, 51100 \\ Reims, France \\ $\left(^{2}\right)$ Unité INSERM 314, 45 Rue Cognacq Jay, Reims, France
}

(Received October 11, 1991; accepted October 21, 1991)

\begin{abstract}
Résumé. - Nous montrons la possibilité d'obtenir des cartes élémentaires semi-quantitatives en microscopie électronique à balayage transmission, à partir de spectres de pertes d'énergie acquis par des méthodes de différence. Ces méthodes notées "méthode FD ou SD" selon que les spectres sont acquis en première ou en seconde différence ont déjà été utilisées pour faire des microanalyses locales d'éléments en très faible concentration. Il avait été montré que cette technique pouvait résoudre les problèmes relatifs à la soustraction du fond continu ainsi que ceux liés à l'inhomogénéité de réponse des diodes dans le cas de la détection parallèle. De plus, cette méthode améliore considérablement la sensibilité de détection des éléments. Nous avons étendu cette technique d'analyse locale pour créer des cartes élémentaires semi-quantitatives. Les études ont été appliquées au matériel biologique cellulaire. Les expériences ont été faites avec un microscope $300 \mathrm{kV}$ STEM (C.M. 30 Philips) couplé à un spectromètre à détection parallèle GATAN et un ordinateur EDAX. Sur un ordinateur hôte interfaçé dans cet ensemble, nous avons développé un logiciel qui assure le contrôle de la sonde STEM sur l'échantillon ainsi que l'acquisition de spectres décalés en énergie et le traitement des données. En comparant des cartes élémentaires obtenues par la méthode conventionnelle (soustraction du fond continu sous le seuil) et la méthode de différence, nous démontrons la validité et les avantages qu'offrent la méthode de différence pour générer des cartes élémentaires multi-éléments semi-quantitatives.
\end{abstract}

\footnotetext{
Abstract. - The feasibility to obtain semi-quantitative elemental maps with a STEM microscope from first or second difference recorded PEELS spectra is demonstrated. These methods called "FD or SD methods" have been already used for the local quantitative analysis of trace elements. It has been shown that it can be a powerful technique to overcome the problems related to the background subtraction and inhomogeneous responses of adjacent diodes in PEELS spectra and also to greatly improve the detectability of the elements. We extended this technique to create semi-quantitative multi-element maps on biological material. The experiments are done with a $300 \mathrm{kV} \mathrm{STEM} \mathrm{(C.M.} 30$ Philips) coupled with a GATAN PEELS and an EDAX computer. A software developed on a host computer allows the control of the STEM probe position and the acquisition of energy shifted EELS spectra for each pixel. By comparing elemental maps obtained, with the conventional method (power law background generation and subtraction above the edge), and with the difference methods we demonstrate both the validity and the advantages offered by FD and SD methods to create multielements semi-quantitative maps.
} 


\section{Introduction.}

The potentiality of electron energy loss spectroscopy (EELS) is now well established for microanalysis [1]. EELS is not only the most sensitive technique to analyse very low $Z$ elements (carbon, nitrogen, oxygen, fluorine) through the K-shell excitation but also important elements like phosphorus, sulfur, chlorine, calcium, iron through the L-shell excitation [2]. The recent use of parallel detection $[3,4]$ has greatly improved the collection efficiency.

The fixed probe analysis can be extended to get a spatially resolved distribution of the elements; this is the basis of the elemental mapping by electron energy loss spectroscopy.

Two different ways can be used to perform it. The first one consists of recording an image with inelastic electrons selected into an energy window. This can be done with a conventional microscope equipped with a Castaing-Henry filter [5] or with an Omega filter [6, 7] or by the addition of a magnetic sector filter placed under the column $[8,9]$. These methods have been called the Electron Spectroscopic Imaging (ESI) by Ottensmeyer [10] or the Electron Filtered Electron Microscopy (EFEM) by Shuman et al. [11]. By this method, while a good lateral resolution can be obtained, the image contrast is not only due to the variation of the local concentration of the element but can be due also to the local thickness variation; it is then necessary to record several energy filtered images and to process them to extract the expected elemental image. A second way consists of scanning an electron probe on the sample and collecting simultaneously the EELS spectra for each probe position. The major advantage of imaging with a scanning probe (for rewiew, see Crewe [12]) is to benefit from the simultaneous collection of all the signals generated by electron scattering. With an analytical scanning transmission electron microscope both the electron energy loss spectrum, the annular dark-field current, and the emitted X-rays, can be recorded at the same time. It is then possible to get a mass-thickness map (D-F STEM Image) and an elemental map by processing the acquired spectra at each pixel.

Several laboratories have combined EELS spectra or X-rays spectra with a computer controlled STEM to produce elemental maps [13-17]. The method generally used was to only keep the interesting part of the raw spectrum for each pixel and to discard the rest of the data collected before the collection for the next pixel. By this method only simple processing can be made. This limitation has been alleviated by collecting the whole spectrum for each pixel in order to record a spectrum-image [18].

Following this idea, we have developed an acquisition system fitted on a C.M. 30 Philips to create elemental maps [19]. The elements we would like to detect can be in low concentration, typically lower than $100 \mathrm{mmole} / \mathrm{kg}$ (biological samples), and then special attention has to be paid to the detection and quantification process.

It is why our system allows not only the recording of one spectrum per pixel but many energy shifted spectra in order to build a multi-spectrum image (MSI), and by calculating first or second difference spectra for each pixel, to build a difference spectrum image (DSI) [20].

From these data different processings can be performed, either by the conventional method or by the so called "difference method". We will describe and compare these two methods and present the advantages of the difference method to create multi-element maps of low concentration elements which are expected or not predicted prior to the acquisition.

\section{PEELS analysis and elemental mapping.}

2.1 The COnVEntional METHOD. - A typical EELS spectrum is composed of a set of edges superimposed to a slowly decreasing background created by the lower energy loss edges and plasmon tails (Fig. 1). To carry out a quantitative elemental analysis, the core edge signal $S(E)$ of 
the chosen element must be extracted from the experimental EELS spectrum $I(E)$ where $E$ is the energy loss. The common way to do this is to model an analytical background function $I_{\mathrm{B}}(E)$. The solution generally accepted consists of finding the best fit between a set of experimental values $I(E)$, for $E<E_{\mathrm{c}}\left(E_{\mathrm{c}}\right.$ is the edge threshold), and a model curve as the power law $I_{\mathrm{B}}(E)=A \cdot E^{-R}$ (Egerton $\left.[21,22]\right)$ by a least square fit method. Then $S(E)$ is obtained by the subtraction $S(E)=I(E)-I_{\mathrm{B}}(E)$ for $E>E_{\mathrm{c}}$. The quantitation is based on the idea that the number of atoms of a chosen element $N_{x}$ in the probed area, is obtained from the equation

$$
N_{x}=\frac{I_{x}(\Delta, \beta)}{I_{\mathrm{i}}(\Delta, \beta)} \cdot \frac{1}{\sigma(\Delta, \beta)}
$$

where $\beta$ is the collection angle, $\Delta$ is the energy window width, and $I_{x}(\Delta, \beta)=\int_{E_{\mathrm{c}}}^{E_{\mathrm{c}}+\Delta} S(E, \beta) \mathrm{d} E$. The partially integrated atomic differential cross-section is written $\sigma(\Delta, \beta)$ and $I_{\mathrm{i}}(\Delta, \beta)$ is the number of electrons measured in the low loss part of the spectrum with the same integration parameters. $\left(I_{\mathbf{i}}(\Delta, \beta)\right.$ is an experimental approximation of the total incident current $\left.I_{\mathbf{i}}\right)$.

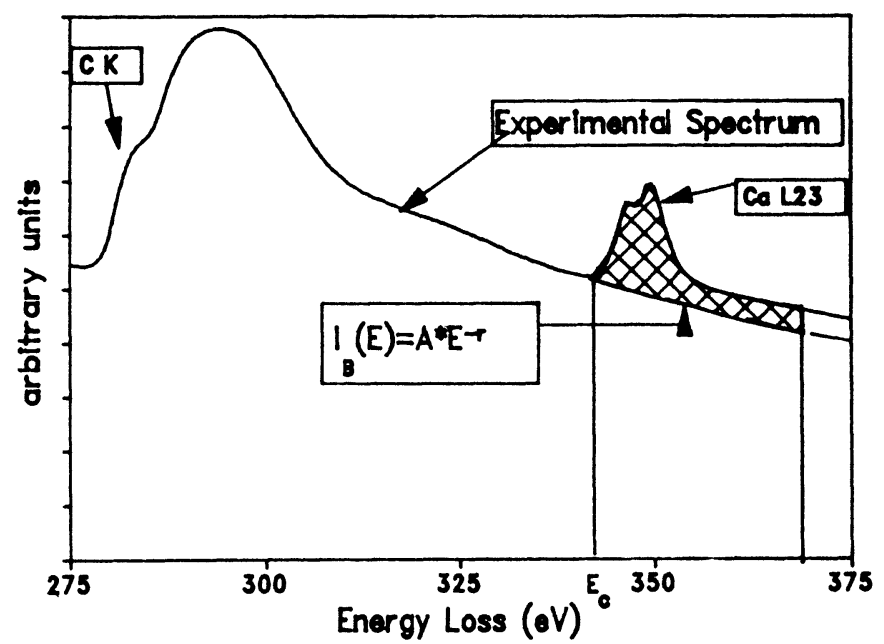

Fig. 1. - The conventional method: a modeled background $I_{\mathrm{B}}(E)=A \cdot E^{-R}$ is extrapolated beneath the edge. The partially integrated signal $I_{x}(\Delta, \beta)=\int_{E_{\mathrm{c}}}^{E_{\mathrm{c}}+\Delta} S(E, \beta) \mathrm{d} E$ is represented by the dashed area.

This method has been currently applied to carry out elemental mapping $[23,24]$. If we are concerned with a semi-quantitative elemental map, due to the linear relation (1), and assuming that $I_{\mathrm{i}}(E, \beta)$ does not vary too much along the different pixels of the map, $N_{x}(p)$, the number of atoms $N_{x}$ probed at each pixel is directly proportional to $I_{x}(\beta, \Delta)$; this is done experimentally by measuring the number of counts in a selected energy window placed just above the edge, minus the counts of the extrapolated background in the same energy window.

It is obvious that the validity of this method is entirely dependant of the quality of the extrapolated background, and when elemental mapping of trace element is performed, many causes of error can be encountered. This is the case when the region used to model the background is too small [25], for instance when overlapping edges have to be separated. 
A second important cause of error frequently encountered is due to the effects of plural scattering. For example, the usual minimum thickness of the ultra-thin section in biological sample is in the range of 60 to 100 nanometers, that is to say in the order of 0.5 to 0.8 carbon plasmon mean free path for $300 \mathrm{keV}$ incident electrons. This is the case, when a calcium quantitative analysis has to be made in a biological sample: the fitting region for the $\mathrm{Ca}_{2} \mathrm{~L}_{3}$ edge is often perturbed by the multiple scattering events.

An other problem may appear when elemental features in the energy loss region situated between 100 and $200 \mathrm{eV}$ have to be analysed (for example phosphorous, sulfur and chlorine). The power law model is no longer valid and other models have to be used to fit the background just after the plasmon losses [26]. False background subtraction can be done and sometimes, negative values of the elemental concentration can be obtained.

Moreover, the parallel detection in spite of increasing the collection efficiency, presents the disadvantage of nonuniform gain due to channel-to-channel variation; this is an additional cause of erroneous fitting.

To overcome these difficulties, Shuman et al. [27] proposed to use the difference method allowing not only to suppress the background, but also to greatly enhance the visibility of very small edges. As an evidence, this method permits to improve the sensibility [28-30] and is well adaptated to the microanalysis of cells [30] (see Figs. 6, 7).

2.2 THE FIRST AND SECOND DIFFERENCE METHOD. - This method requires the acquisition of two or three energy shifted spectra, consequently increasing the acquisition time. The voltage shift $\delta$ between two consecutive spectra must be properly chosen to remove the background beneath the edge while avoiding the deterioration of the signal to noise ratio. A $\delta$ values of 4 to $8 \mathrm{eV}$ [28-30] is generally used and can be extended up to $15 \mathrm{eV}$ [32]. Noticing $X_{1}(E), \ldots X_{3}(E)$ the different voltage shifted spectra ( $\delta$ in $\mathrm{eV}$ ), first difference and second difference spectra are respectively calculated as:

$$
\begin{aligned}
& \mathrm{FD}(E)=X(E)-X(E+\delta) \\
& \operatorname{SD}(E)=2 . X(E)-X(E+\delta)-X(E-\delta)
\end{aligned}
$$

In the case of low elemental concentration this method not only suppresses the background but also can reduce the effects due to the inhomogeneous response of the detector array. This can be demonstrated as follows:

Let us write $X(E)=(B(E)+S(E))$

Where $B(E), S(E)$, are respectively the background and the signal. The measured intensity for the channel $N$ is:

$X(N)=(B(E)+S(E)) \cdot R(N)$ where $R(N)$ is the detector response for the channel $N$, and $E=\alpha \cdot N+E_{0}$

$\alpha$ is the spectrometer dispersion coefficient and $E_{0}$ is the voltage offset.

With $B(E)=A \cdot E^{-r}$, we have $B(E+\delta)=A \cdot(E+\delta)^{-r}$ which can be written in a first order approximation, assuming $\delta<<$, as:

$$
\text { and } \quad \begin{aligned}
B(E+\delta) & =A \cdot E^{-r} \cdot(1-r . \delta / E) \\
B(E-\delta) & =A \cdot E^{-r} \cdot(1+r . \delta / E)
\end{aligned}
$$

Then a second difference spectrum is given by:

$$
\mathrm{SD}(N)=2 \cdot X_{2}(N)-X_{1}(N)-X_{3}(N)
$$


that is to say:

$$
\begin{aligned}
\mathrm{SD}(N)= & R(N) \cdot[2 \cdot B(E)-B(E+\delta)-B(E-\delta)]+ \\
& R(N) \cdot[2 \cdot S(E)-S(E+\delta)-S(E-\delta)]
\end{aligned}
$$

From relations (3) we can see that the difference in the first bracket of the relation (4) cancels. Then while the background is entirely suppressed, the artefacts introduced by $R(N)$ are greatly reduced. The response of the detector array affects only the signal difference which corresponds to a small modification in the shape of the signal difference.

The process of quantitation:

In these cases, the spectrum edges shapes are not preserved and a new method of quantitation has been derived. $\mathrm{FD}(E)$ or $\mathrm{SD}(E)$ are fitted with a weighted sum of difference reference spectra recorded in the same conditions.

This can be described mathematically as follows (see [28] for instance). Firstly starting from a raw spectrum, the influence of multiple scattering events due to the sample thickness $t$ has to be taken into account by the following relation (5).

$$
X(E, t)=X(E, 0) *\left\{\mathrm{e}^{-t / \lambda} \cdot\left[\delta(E)+\Sigma(t / \lambda)^{n} \cdot \operatorname{LL}(E)^{* n} / n !\right]\right\}
$$

where $X(E, 0)$ represents the ideal spectrum corresponding to a very thin sample $(t / \lambda<0.1), *$ means a convolution product, $n$ is the multiple scattering event. ${ }^{* n}$ indicates $n$ repeated selfconvolutions and $\operatorname{LL}(E)$ is the low loss single scattering distribution.

Assuming that the thickness is not too large $X(E, t)$ can be written for example by the simplified expression (6):

$$
X(E, t)=X(E, 0)+a_{1} \cdot X(E, 0) * \operatorname{LL}(E)+a_{2} \cdot X(E, 0) * \operatorname{LL}(E) * \operatorname{LL}(E)
$$

$a_{1}, a_{2}$ are two coefficients which should be the two first coefficients of the Poisson distribution.

However, the energy loss spectrum $X(E, 0)$ can be written as a weighted sum of reference spectra $R_{j}(E, 0)$ :

$$
X(E, 0)=k_{1} \cdot R_{1}(E, 0)+\ldots .+k_{j} \cdot R_{j}(E, 0)
$$

In a biological sample where carbon is predominant and the interesting elements are in low concentration, the convolution effect described before, affects essentially the major term of this sum, that is to say the carbon spectrum $R_{1}(E, 0)$. Then $X(E, t)$ can be written more simply as (8):

$$
\begin{aligned}
X(E, t)= & k_{1} \cdot\left(R_{1}(E, 0)+a_{1} \cdot R_{1}(E, 0) * \mathrm{LL}(E)+a_{2} \cdot R_{1}(E, 0) * \mathrm{LL}(E) * \operatorname{LL}(E)\right) \\
& +k_{2} \cdot R_{2}(E, 0)+\ldots \ldots+k_{j} \cdot R_{j}(E, 0)
\end{aligned}
$$

Due to the linear properties of the derivation, a similar relation can be written for the first difference spectrum as:

$$
\begin{aligned}
\mathrm{FD}(E, t)= & k_{1} \cdot\left(R_{1}^{\prime}(E, 0)+a_{1} \cdot R_{1}^{\prime}(E, 0) * \mathrm{LL}(E)+a_{2} \cdot R_{1}^{\prime}(E, 0) * \mathrm{LL}(E) * \mathrm{LL}(E)\right) \\
& +k_{2} \cdot R_{2}^{\prime}(E, 0)+\ldots \ldots+k_{j} \cdot R_{j}^{\prime}(E, 0)
\end{aligned}
$$

where $R_{j}^{\prime}(E, 0)$ denotes a reference spectrum acquired in the first difference mode. A second difference spectrum will follow a same relation.

To determine the relative concentration of different elements in an unknown sample, the $a_{j}$ and $k_{j}$ coefficients have to be calculated by a multiple least square fitting (M.L.S.) between the 
experimental acquired spectrum and a weighted sum of reference spectra, following the relations (8), (9). This method can be applied either starting from a raw spectrum or from first or second difference acquired spectra $[2,28,31,33]$ because in each case the coefficients are the same. The CURFIT algorithm [34] can be used to perform the M.L.S. procedure.

The concentration ratio between two elements will be given by (10):

$$
C_{i} / C_{j}=\left(k_{i} / k_{j}\right) \cdot\left(n_{i} / n_{j}\right) \cdot\left(\sigma_{j} / \sigma_{i}\right)
$$

where $n_{i}$ and $n_{j}$ are the numbers of electrons counted into a fixed energy window $\Delta$ in the edges of the reference spectra $i$ and $j$, and $\sigma_{i}, \sigma_{j}$ are the corresponding partial cross-sections evaluated for the same energy window and a fixed collection angle $\beta$.

If we are concerned by quantitative elemental mapping, this whole procedure must be repeated for each pixel.

Sometimes, for a semi-quantitative analysis, only the variation of the local concentration of an element is a pertinent information; then the determination of the variation of the $k_{j}$ value for each pixel may be sufficient; then, using difference spectra (FD or SD), the M.L.S. procedure can be performed on a reduced part of the spectrum (energy window). In this selected energy window, the elemental feature appears as an oscillation above an expected mean zero value. The amplitude of this oscillation is proportional to the $k_{j}$ factor as shown in the relations (8) and (9), and is also proportional to the number of atoms in the probed area as indicated in the relation (10).

Assuming that the general aspect of this oscillation does not change too much with the local variation of the element concentration (Fig. 2), it can be considered as a fingerprint of the element. A semi-quantitative analysis consists then in the determination of a scaling factor between experimental and reference fingerprints obtained in the same acquisition conditions.

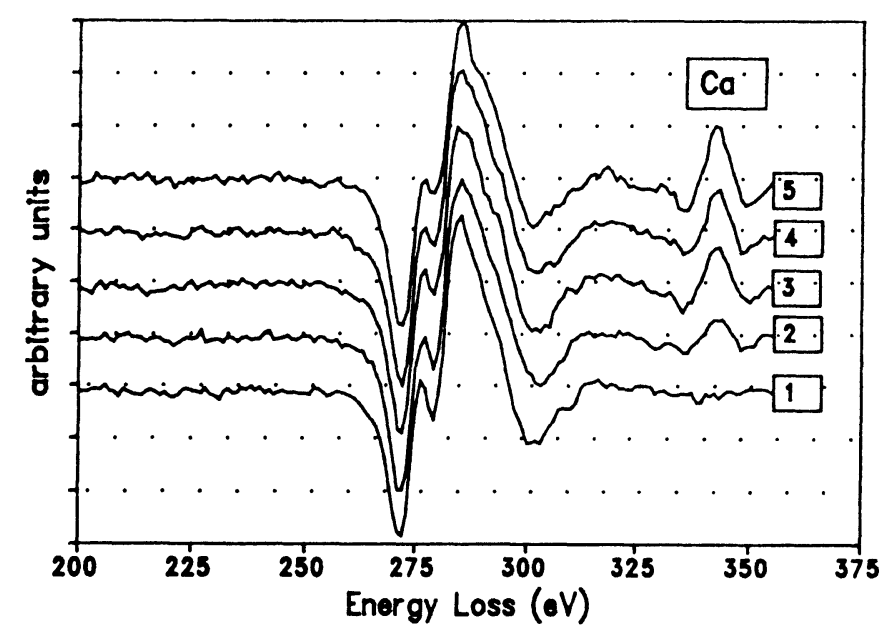

Fig. 2. - Five Second Difference spectra showing the carbon $\mathrm{K}$ edge and calcium $\mathrm{L}_{23}$ edge for different calcium concentration. The second difference spectra were obtained from three raw spectra shifted by $4 \mathrm{eV}$.

As a rough approximation, we can consider that the amplitude of the oscillation in the window (the maximum minus the minium) gives an acceptable approach of the expected scaling factor or $k_{j}$ coefficient. 
This quick process is easy to perform, but as a precaution, it is better to add to this procedure an automatic background subtraction in order to get a constant baseline inside the window [35]. Indeed, depending of the energy position of the edge, even for SD spectra the background removal is not always perfect. When the edge is placed at energy between 100 and $200 \mathrm{eV}$, the elemental oscillations are always superimposed on a varying base-line and it can be extracted from the second difference spectrum by subtracting a mean function in the selected energy window; we approximate this mean function as a linear function calculated on the chosen energy window (Fig. 9d).

By this method it is quick to perform semi-quantitative multi-element maps starting from a second difference spectrum image and selecting different windows in this difference spectrum image (Fig. 3). We will show that it is possible to get maps of elements like phosphorous, calcium, at the level of concentration commonly encoutered in biological sample ( $<100 \mathrm{mmole} / \mathrm{kg})$. Obviously other elements like oxygen, nitrogen, iron etc. can also be mapped.

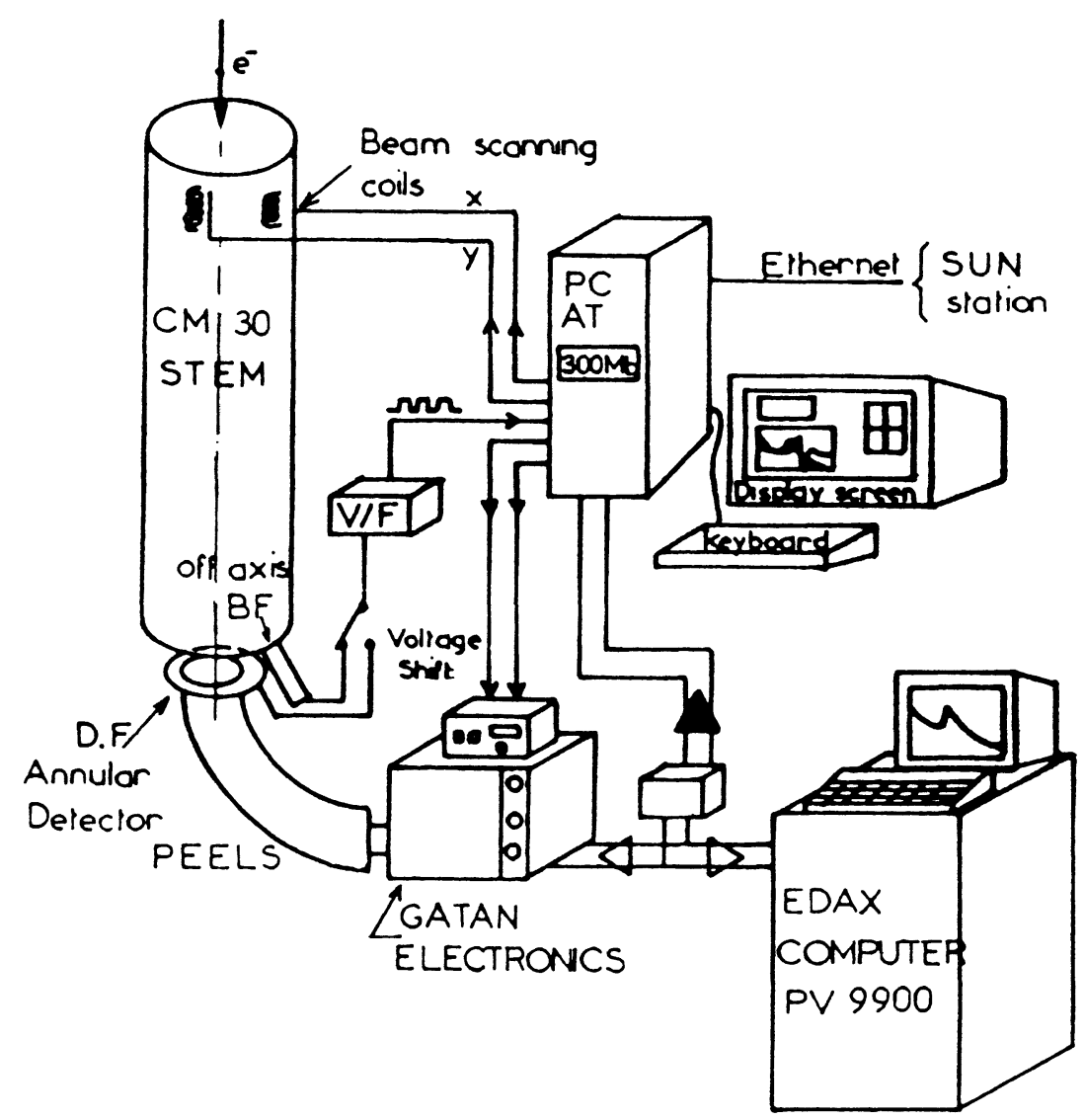

Fig. 3. - The host computer interfaced with the C.M. 30 Philips. The interface hardware controls the probe scanning via two D/A converters. EELS spectra (1024 data) and shield current are read via 18 lines interfaced with the bus connecting the GATAN electronics to the EDAX computer. The annular dark-field current is recorded via a V/F converter. A D/A converter connected to the GATAN electronics allows the controls of the voltage applied to the spectrometer drift tube. Voltage shifted raw spectra can be recorded. A multifunction software implemented on the host computer allows data acquisition and processing. 


\section{Material and method.}

3.1 ACQUISITION. - The experiments presented in this article have been performed with a $300 \mathrm{kV}$ TEM and STEM (C.M. 30 Philips). This electron microscope is coupled with a parallel electron energy loss spectrometer (GATAN PEELS 666). The energy loss spectrum is read from a $1 \mathrm{D}$ photodiode array as a series of one or more detector integrations. The minimum integration time is $24 \mathrm{~ms}$, due to the photodiode array readout time. The sensitivity of the photodiode is about one count per 40 impinging electrons. The portion of the spectrum not falling on the photodiode array is intercepted on a shield coupled with a nanoampermeter giving the so called shield current.

An annular on-axis dark-field detector and near-axis bright-field and dark-field detectors are also available. They are placed just above the parallel EEL spectrometer. Then it is possible to get almost all the electrons simultaneously transmitted through a sample from a probed area.

Elemental maps are obtained by scanning the electron probe on the sample while recording the energy loss spectrum for each pixel. In order to benefit from all the signals collected we have developed a multifunction software allowing control of the acquisition and processing of these data. This software is implemented on a host computer (PC AT 386) interfaced with the analytical microscope (Fig. 3).

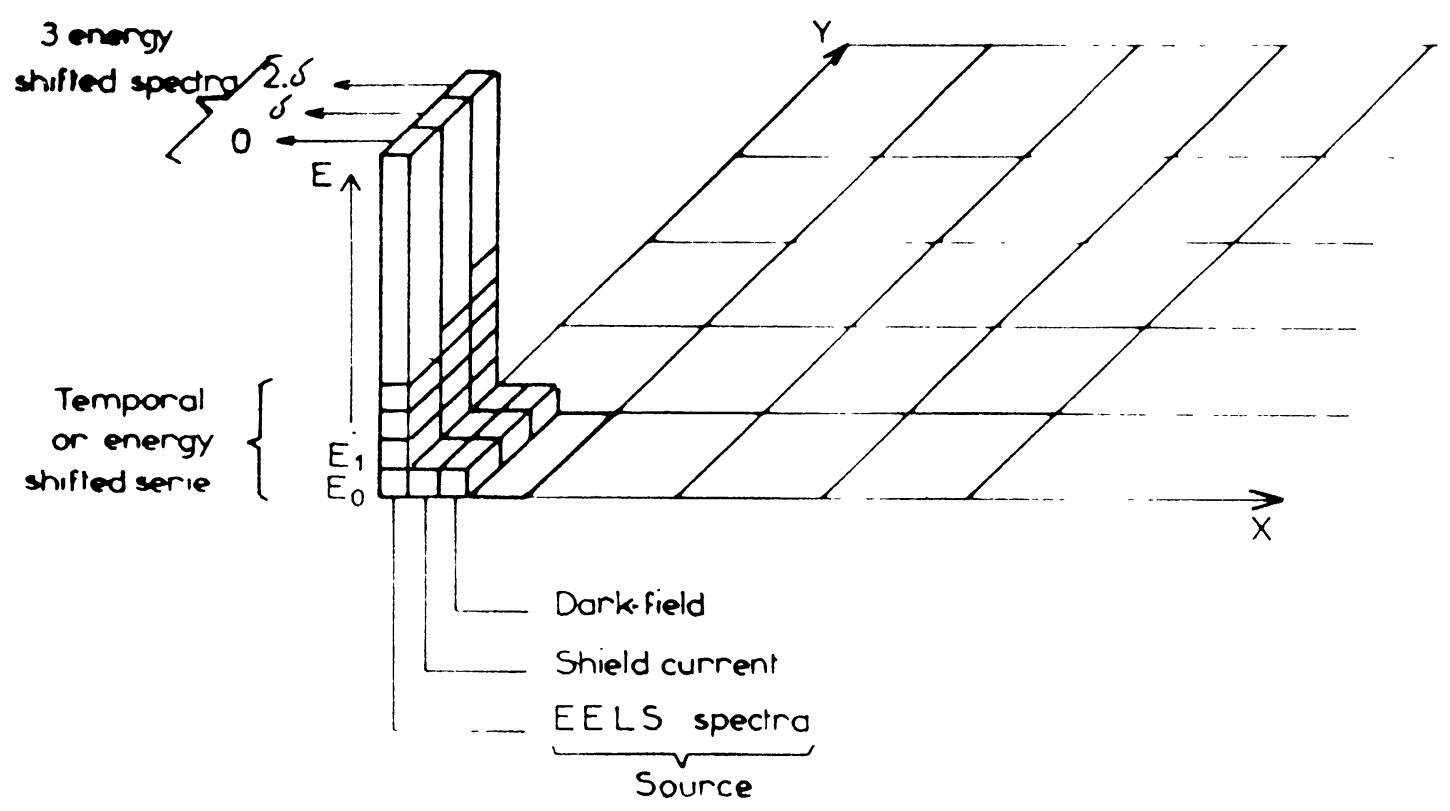

Fig. 4. - The multiple spectrum image (MSI). For each pixel, the data recorded are arranged in series and blocks. The series contain the voltage shifted raw spectra (1024 data) and the blocks contain the shield current and the dark-field current. This file can be read along vertical lines to get the different raw spectra, or along horizontal sections to get energy filtered images. The difference spectrum image (DSI) is built by replacing $\mathrm{FD}(E)$ or $\mathrm{SD}(E)$ spectra in place of the raw spectra for each pixel.

A complete description of this software and hardware will be published elsewhere. We only present some of its specifications in this article. As we can see in the figure 3 the interface hardware 
controls the scanning of the probe via a digital to analog converter. It allows the acquisition of the EELS spectrum (raw spectrum) and the shield current via 18 line interfaced with the bus connecting the GATAN electronics to the EDAX computer. The annular dark-field current is also recorded for each pixel via a voltage to frequency converter.

To start an acquisition, the EDAX computer operates in the permanent mode of acquisition with a chosen dwell time. The software insures the synchronism between the EDAX and the host computer. The operations are unfolded following this sketch: $X$ and $Y$ probe shifting, raw spectrum acquisition (1024 data), shield current and dark-field current readout for each pixel. In order to create elemental maps in the first or second difference mode we have to record two or three voltage shifted raw spectra for each pixel. This can be done via the digital to analog converter connected to the GATAN electronics and controlling the voltage applied to the spectrometer drift tube. To avoid the memory effects on the diode array, the voltage shift is applied after a line scan in place of doing it for a fixed probe position.

At the end of the acquisition the whole data are stored in a multiple spectrum image (MSI) (see Fig. 4). For each pixel the data recorded are arranged in series and blocks; the series contain the voltage shifted raw spectra and the blocks contain the shield and dark-field currents.

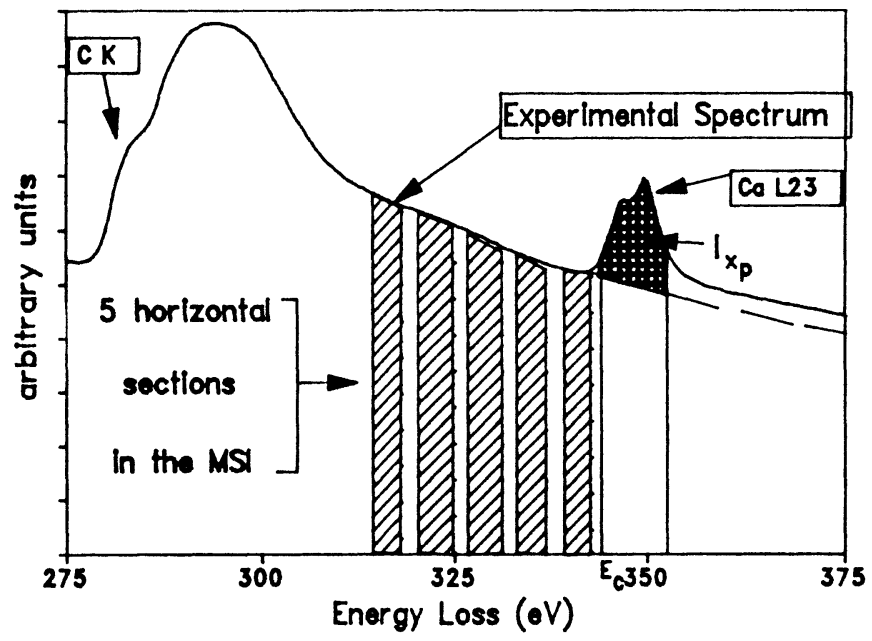

Fig. 5. - The conventional method. Five windows are selected below the edge by placing cursors on a displayed sectrum chosen inside the MSI. These windows correspond to horizontal sections (energy width $\Delta$ ) in the MSI. The background $I_{\mathrm{B} p}(E)=A_{p} \cdot E^{-R}$ calculated by a least square fit method is extrapolated beneath the edge $\left(E>E_{\mathrm{c}}\right)$ and subtracted from the raw spectrum in the sixth energy window, for each pixel $p$. The $I_{x_{p}}$ values are obtained by integrating the data contained above the background inside this last selected energy window.

3.2 Processing. - Processing MSI allows the creation of elemental maps either with the conventional method or with the first or second difference method described in section 2.

In the conventional method the background has to be extrapolated beneath the edge $\left(E>E_{\mathrm{c}}\right)$ and subtracted from the raw spectrum in a energy window for each pixel $p$. From a set of experimental values $I_{p}(E)$, and for $E<E_{\mathrm{c}}$, a model curve as the power law $I_{\mathrm{B} p}(E)=A_{p} . E^{-R}$ is calculated by a least square fit method. The $I_{p}(E)$ values are obtained by integrating the data 
contained into selected energy window $\Delta$ (corresponding to horizontal sections in the MSI); i.e. $I_{p}(E, \Delta, \beta)$. This is done experimentally by selecting windows on a displayed raw spectrum chosen among a spectra series stored in the MSI (see Fig. 5). Generally six windows are selected, five below the edge threshold and the sixth above the edge.

Then $I_{x_{p}}(\Delta, \beta)$ is obtained ffrom the relation (11):

$$
I_{x_{p}}(\Delta, \beta)=I_{p}(E, \Delta, \beta)-I_{\mathrm{B} p}(E, \Delta \beta) \text { for } E>E_{\mathrm{c}} .
$$

The semi-quantitative elemental map is then directly obtained from these $I_{x_{p}}(\Delta, \beta)$. A quantitative map should be obtained by using the relation (1).

In the difference method, the processing must be made in two steps. Starting from a series of energy shifted raw spectra, the FD or SD spectra (Figs. 6, 7) are calculated for each pixel by applying the relations (2). A new spectrum image called "difference spectrum image" (DSI) is then built. By the method described in the paragraph 2.2 a semi-quantitative multi-element image may be made quickly. The elements we want to map are chosen by selecting their characteristic features in a displayed difference spectrum chosen among the DSI. This is done experimentally by positionning the windows on the displayed spectrum and fitting their widths so that the elemental features are entirely bracketted. The amplitude of the oscillations gives the semi-quantitative map of the selected element (Fig. 8b, Fig. 9d).

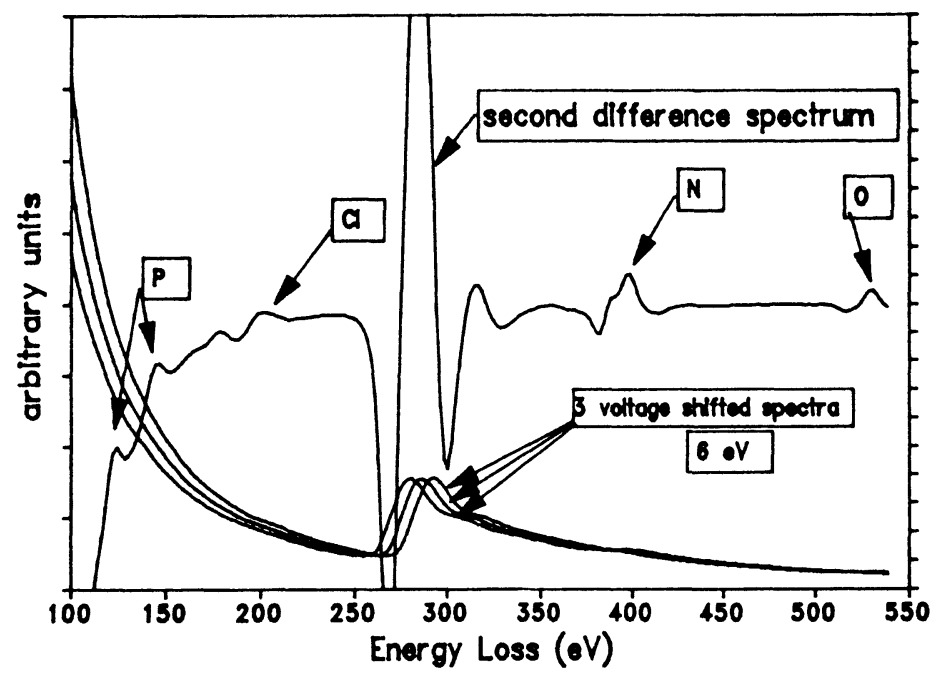

Fig. 6. - The difference method. A second difference spectrum obtained from three voltage shifted $(6 \mathrm{eV})$ spectra. The sample is a cell nucleus. The diameter of the probed area is $30 \mathrm{~nm}$. The energy of the incident electrons is $300 \mathrm{keV}$. The collection angle is $27 \mathrm{mrd}$. The dwell time is $0.5 \mathrm{~s}$ for each raw spectrum. Phosphorous, carbon, nitrogen, oxygen contained in the biological sample and chlorine arising from the embedding medium are clearly revealed in the second difference spectum. 


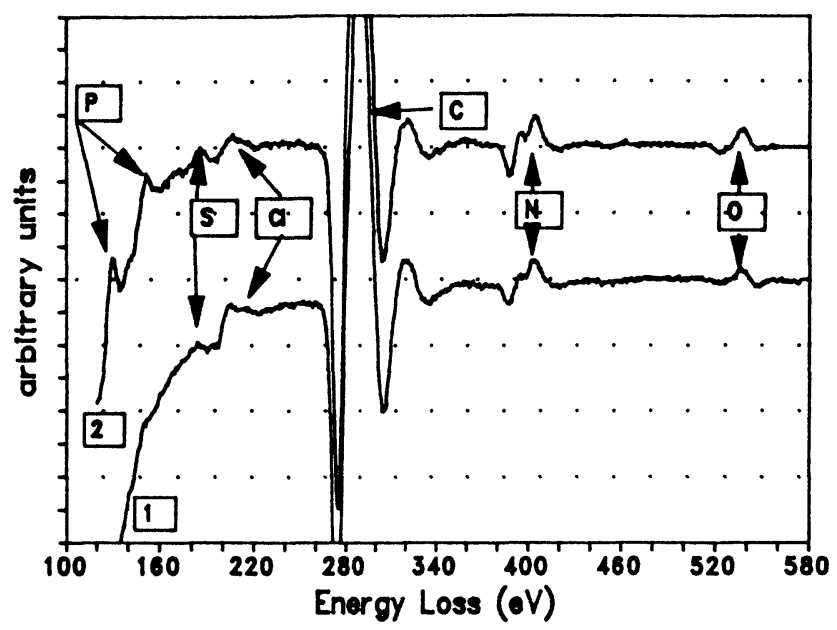

Fig. 7. - Two second difference spectra recorded from two different probed area in a cell. On the SD spectra (1 and 2) the electron beam probes respectively the cytoplasm and the nucleous. The carbon, nitrogen, oxygen second difference $\mathrm{K}$ edges have approximately the same amplitude in the two curves. The chlorine second difference $\mathrm{L}_{23}$ edge is mainly due to the embedding resin. Phosphorous is well expressed in the curve 2 corresponding to nucleotides (e.g. DNA or ATP).

Experimental parameters: Incident electrons energy: $300 \mathrm{keV}$; collection angle: $27 \mathrm{mrd}$; probe diameter: $30 \mathrm{~nm}$; dwell time: $3 * 0.5 \mathrm{~s}$.

\section{Experimental results.}

Different elemental maps obtained either with the conventional method or using the difference method are displayed in Figures 8 and 9.

In figure 8, we compare two calcium maps obtained on the same specimen area with the conventional method ( $\mathrm{Ca} \mathrm{CM}$ map) and with the difference method using first difference spectra (Ca FD map).

The sample is hydroxyapatite crystals contained in a vacuol of a macrophagis section. The data recordings are made in the STEM mode. The collection angle is $27 \mathrm{mrd}$. The image size is $64 * 64$ and the electron probe diameter is $2.4 \mathrm{~nm}$. The analysed area is $160 \mathrm{~nm}$. square. Two energy shifted $(8 \mathrm{eV})$ raw spectra (1024 data) are recorded for each pixel. The spectrometer energy resolution is $1 \mathrm{eV} / \mathrm{channel}$. The dwell time for each spectrum is $100 \mathrm{~ms}$. To reduce the size of the data file (MSI), each raw spectrum is compressed into 256 data during the storage step.

The Ca CM map is performed by the conventional method described in the paragraph 3; it is a semi-quantitative map. The Ca FD map is achieved by measuring the amplitude of the oscillation in an energy window bracketting the Ca feature in the first difference spectra (Fig. 8b).

As it is shown, the two maps are similar; this is confirmed by a two dimensional histogram (Scatter in Fig. 8) comparing the intensities for each pixel in the two Ca maps (vertical axis Ca FD data, horizontal axis $\mathrm{Ca} C M$ data). This is the proof of the validity of the difference method. An oxygen map (O FD) obtained by the difference method is also shown in figure 8.

In the next experiment (Fig. 9), we would like to demonstrate the advantages offered by the difference method to create multi-element maps. As discussed before, the main problem encountered in the conventional method arises from the background extrapolation beneath the edge. This problem is specially evident when the characteristic edge lies in the 100 to $200 \mathrm{eV}$ energy loss 
a)

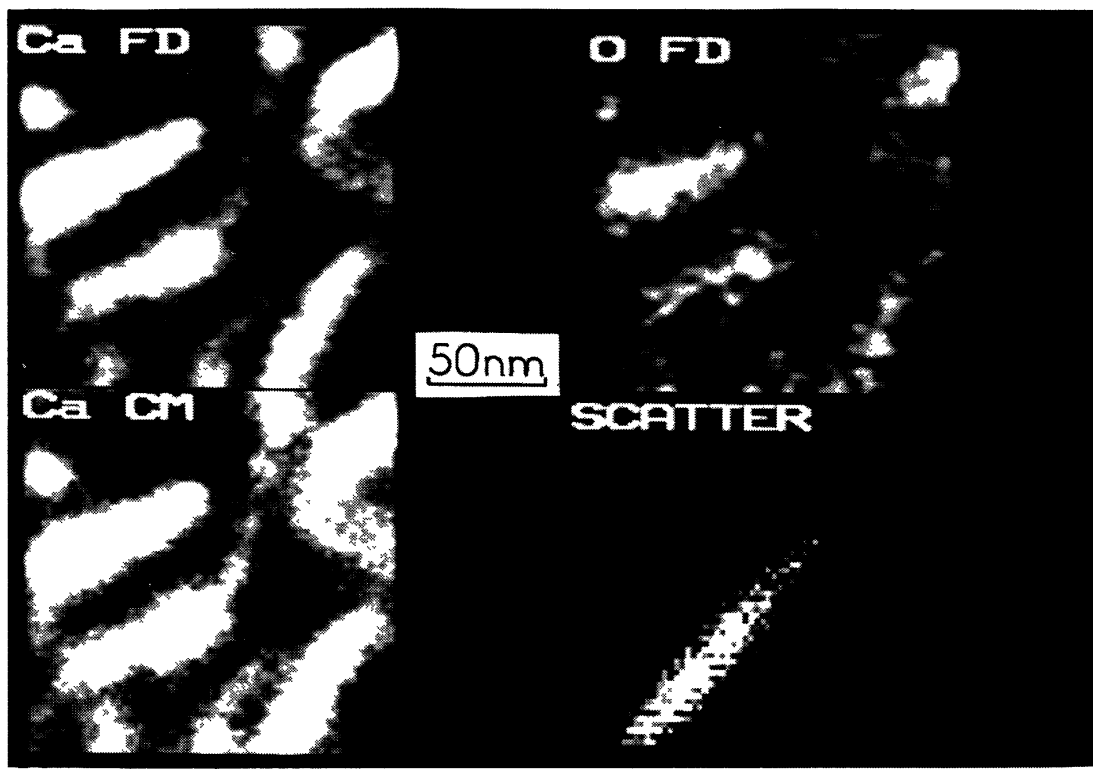

b)

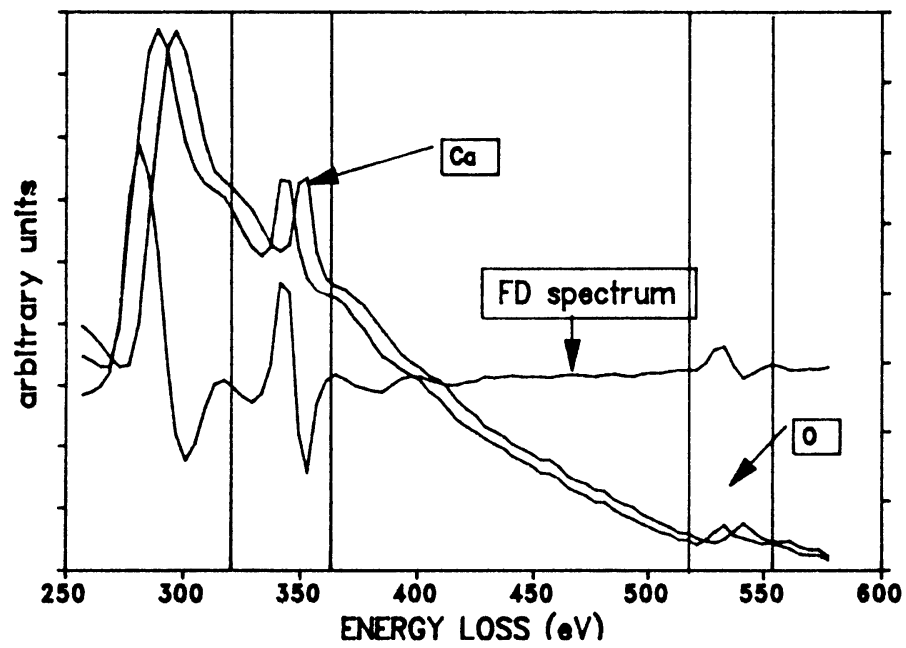

Fig. 8. - a) Two calcium maps of hydroxyapatite crystals in a vacuol of a macrophagis section performed on the same area; the first one with the conventional method ( $\mathrm{Ca} \mathrm{CM})$ and the second with the difference method using a first difference spectrum (Ca FD). The semi quantitative (Ca FD) map is obtained by measuring the amplitude of the oscillation in an energy window bracketting the Ca feature in the first difference spectra (Fig. 8b). A perfect correlation between these two maps is confirmed by the two dimensional histogram (Scatter). An oxygen map (O FD) obtained in the same condition as the Ca FD is shown. The images size is $64 * 64$. The electron probe diameter is 2.4 nanometres. The analysed area is 160 square nanometres. Two energy shifted ( $8 \mathrm{ev}$ ) raw sectra (1024 data) are acquired for each pixel. The spectrometer energy resolution is $1 \mathrm{eV} /$ channel. The dwell time for each spectrum is $100 \mathrm{~ms}$. 

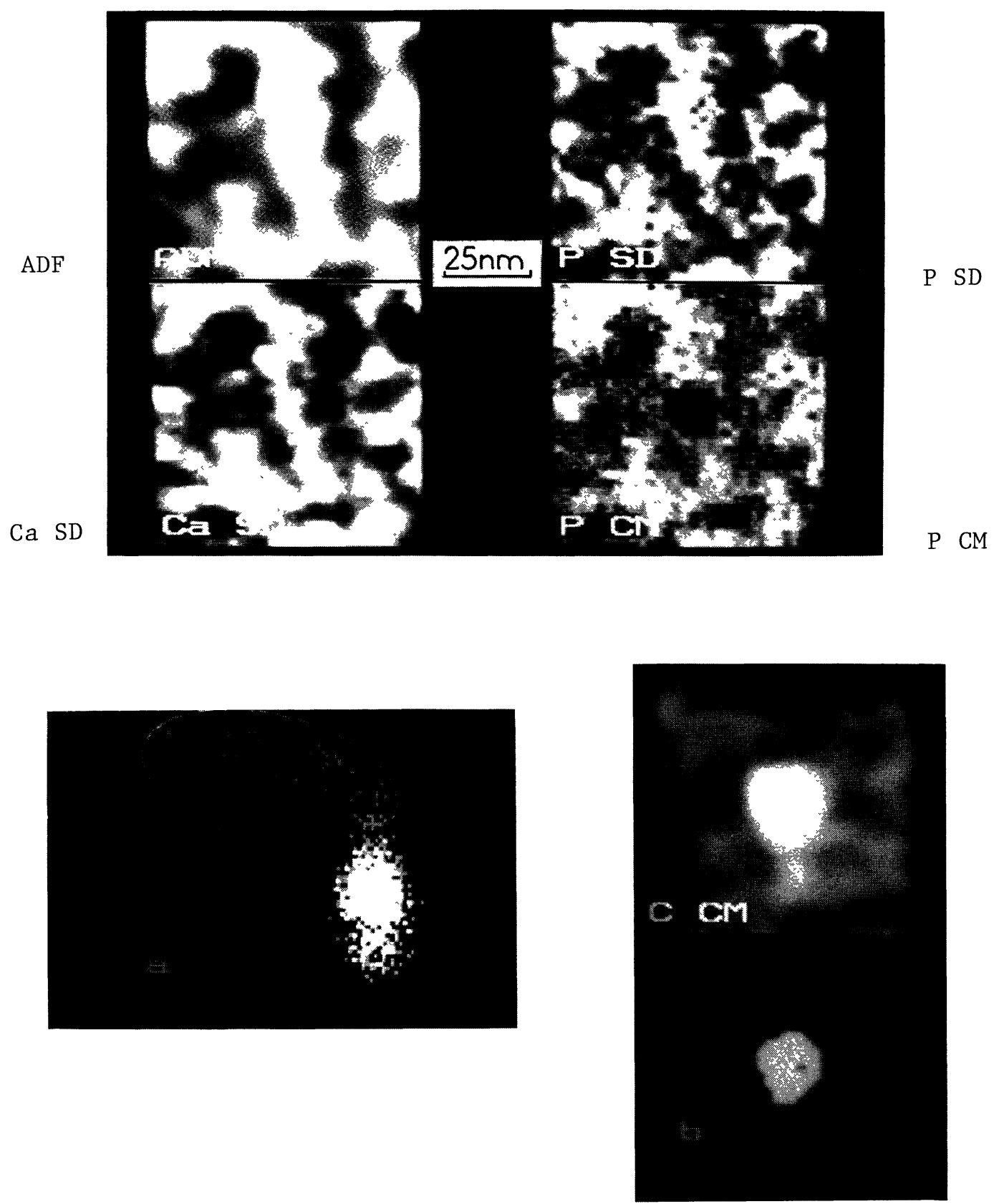

Fig. 9. - Annular dark-field image (ADF), $\mathrm{P}$ and $\mathrm{C}$ maps obtained by the conventional method (P CM, $\mathrm{C} \mathrm{CM}$ ), and $\mathrm{Ca}$ and $\mathrm{P}$ maps obtained by the second difference method (Ca SD, P SD) of hydroxyapatite crystals with a carbon contamination spot deposited at the center of the mapped area. One of the second difference spectra obtained from 3 energy shifted $(4 \mathrm{eV})$ raw spectra is shown in Fig $9 \mathrm{~d}$. The two dimensional histogram (a) between the carbon map (vertical axis) and the P CM map (horizontal axis). Binary image built by satisfying the histogram constraint (circled area in (a)). The image size is $32 * 32$ and the electron probe diameter is $2.4 \mathrm{~nm}$. The analysed area is $80 \mathrm{~nm}^{2}$. The spectrometer energy resolution is $1 \mathrm{eV} / \mathrm{channel}$. The dwell time for each spectrum is $100 \mathrm{~ms}$. 


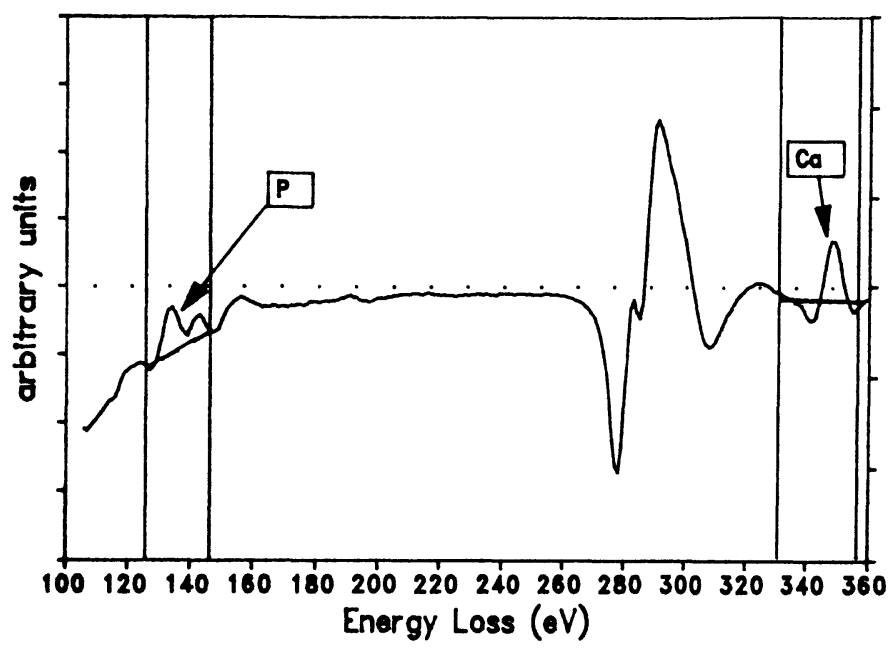

d)

Fig. 9. - (continued)

region; this is the case when phosphorous has to be mapped by using the $\mathrm{L}_{23}$ edge. In this case, if the local specimen thickness becomes large $(t / \lambda>1)$, the power law model is no longer valid, and spurious effects like a negative value of the net recorded signal minus computed background may appear.

This effect is shown in figure 9 where we compare phosphorous maps obtained by the conventional and by the difference method (P CM map and P SD map). In order to improve the background subtraction in the difference spectra we used second difference spectra (Fig. 9). Three energy shifted ( $4 \mathrm{eV}$ ) raw spectra (256 data) are acquired for each pixel. The spectrometer energy resolution is $1 \mathrm{eV} / \mathrm{channel}$. The dwell time for each spectrum is $100 \mathrm{~ms}$. The image size is $32 * 32$ and the electron probe diameter is $2.4 \mathrm{~nm}$. The analysed area is $80 \mathrm{~nm}^{2}$.

The test sample is hydroxyapatite crystals (as in Fig. 8). A carbon coating spot has been deposited at the center of the mapped area by contamination.

The sample morphology is shown on the annular dark field image (ADF). A calcium map (Ca SD) obtained in the second difference mode looks like the ADF image.

Let us compare now the phosphorous maps obtained by the two methods (P SD map and P CM map). A black area corresponding to negative measured values of phosphorous concentration appears at the center of the P CM map while phosphorous is well revealed in this area on the second difference phosphorous map (P SD).

This artefact comes from the local thickness variation in the sample as it is confirmed by the carbon map (C CM). A two dimensional histogram (Fig. 9a) between the carbon map (vertical axis) and the P CM map (horizontal axis) shows the intensity distributions in these maps; phosphorous is not detected in the P CM map where carbon concentration is large. By selecting the cloud corresponding to a large carbon concentration (circled area Fig. 9a) on the two dimensional histogram, a binary image satisfying the histogram constraint can be built. As it is shown (Fig. 9b) this binary image corresponds to the carbon contamination spot; this a confirmation of the hypothesis discussed before. 


\section{Conclusion.}

The goal of this article was to present a quick method to produce multi-element maps with a conventional TEM-STEM microscope taking benefit of the advantage offered by the difference methods in parallel electron energy loss spectroscopy. We first demonstrate the validity of this method by comparing it with the conventional method on a test sample and we prove that the problems like the mapping of phosphorous can be solved. This semi-quantitative approach can be extended in order to get quantitative elemental maps by using MLS method.

\section{Acknowledgements.}

The authors would like to thank M. Ménager and J. Hinnrasky for specimen preparations.

\section{References}

[1] ISAACSON M.S. and JOHNSON D., Ultramicroscopy 1 (1975) 33-52.

[2] LEAPMAN R.D. and HUNT J.A., Microsc. Microanal. Microstruct. 2 (1991) 231-234.

[3] SHUMAN H., Ultramicroscopy 6 (1981) 163-168.

[4] KRIVANEK O.L., AHN C.C. and KEENEY R.B., Ultramicroscopy 22 (1987) 103-116.

[5] Castaing R. and Henry L., C.R. Acad. Sci. Paris 255 (1962) 76.

[6] ZANCHI G., PEREZ J.P., SEVELY J., Optik 43 (1975) 495.

[7] ZANCHI G., SEVELY J., JOUfFREY B., J. Microsc. Spectrosc. Electron. 2 (1977) 95-104.

[8] Shuman H., SOMLYo A.P., in Analytical electron microscopy, Ed. R.H. Geiss (San Francisco Press, 1981) p. 202.

[9] KRIVANEK O.L., Gubens A.J., DellBy N., Microsc. Microanal. Microstrc. 2 (1991) 315.

[10] OTTENSMEYER F.P., J. Ultrastruct. Res. 88 (1984) 121-134.

[11] Shuman H., CHANG C.F., SOMLYO A.P., Ultramicroscopy 19 (1986) 121-134.

[12] CREWE A.V., Science 221 (1983) 325-330.

[13] Jeanguillaume C., Colliex C., TREBbia P., Ultramicroscopy 3 (1978) 137-142.

[14] Jeanguillaume C., Tence M., Trebbia P. and Colliex C., in Scanning Electron Microscopy 2, O.J. Ohari Ed. (SEM, AMF O'HARE, il 1983) p. 745.

[15] COllieX C., JEANGUILlaUme C. and MORY C., J. Ultrastruct. Res. 88 (1984) 177.

[16] SOMLYO A.P., J. Ultrastruct. Res. 88 (1984) 135.

[17] FIORI C.E., LEAPMAN R.D., SWYT C.R. and ANDREWS S.B., Ultramicroscopy 24 (1988) 237.

[18] JeANGUILlaume C., COllieX C., Ultramicroscopy 28 (1989) 252.

[19] Thomas X., Balossier G., Wagner D., Marin P., Bonhomme P., in Proc. XII ${ }^{\text {th }}$ Int. Cong. E.M. (San Francisco Press) 2 (1990) 30.

[20] Balossier G., Thomas X., Michel J., Bonhomme P., Proceedings C.F.I.M.E. Barcelone (1991) 298.

[21] EGERTON R.F., Philos. Mag. 31 (1975) 199-205.

[22] EgERTON R.F., in Electron Energy-Loss Spectroscopy in The Electron Microscope, (Plenum Press, New York, 1986).

[23] Janguillaume C., Trebila P., Colliex C., Ultramicroscopy 3 (1978) 237.

[24] Bonnet N., COlliex C., MORY C., TENCE M., Scanning Mircrosc. Supl. 2 (1988) 351.

[25] TREBBiA P. and MANOUBI T., Ultramicroscopy 28 (1989) 266-276.

[26] Tenaillau H. and MarTin J.M., Proceedings C.F.I.M.E. Barcelone (1991) pp. 303-304.

[27] Shuman H. and KRUIT P., Rev. Sci. Instrum. 56 (1985) 231-239.

[28] SHUMAN H. and SOMLYO A.P., Ultramicroscopy 21 (1987) 23-32.

[29] KRIVANeK O.L., MORY C., TenCE M., CollieX C., Microsc., Microanal, Microstruct. 2 (1991) 257-267.

[30] LEAPMAN R.D. and SwYT C.R., in Microbeam Analysis, P.E. Russel Ed. (San Franciscco Press, 1989) 89-93.

[31] LEAPMAN R.D. and SWYT C.R., Ultramicroscopy 26 (1988) 393-404. 
[32] WANG Y.Y., RUOYA H., ZHIFENG S. and SOMLYo A., Biological Electron Energy Loss Spectroscopy: Background Subtraction and Optimized Quantitation of Phosphorous and other Elements in the Low-loss Region (to be published).

[33] Wagner D., Balossier G., Thomas X., Michel J., Bonhomme P., Proceedings C.F.I.M.E. Barcelone (1991) p. 259.

[34] BEvingTON P.R., in Data Reduction and Error Analysis for the Physical Science (M.C. Graw Hill, 1969) pp. 237-247.

[35] KUNDMANN M. and KRIVANEK O.L., Microsc. Microanal. Microstruct. 2 (1991) 245-256. 